\title{
Ferramentas Pedagógicas em Ambientes Virtuais de Aprendizagem e a Taxonomia Revisada de Bloom
}

\author{
Selma Márcia Pontes Teixeira Rocha ${ }^{1}$, Rommel Wladimir de Lima ${ }^{1}$ \\ ${ }^{1}$ Programa de Pós-Graduação em Ciência da Computação - PPgCC \\ Universidade do Estado do Rio Grande do Norte - UERN \\ Universidade Federal Rural do Semiárido - UFERSA \\ Laboratório de Redes e Sistemas Distribuídos - LORDI \\ BR 110 - Km 46 - Bairro Costa e Silva, 59.625-620, Mossoró - RN, Brasil \\ \{selmapontes@yahoo.com.br, rommelwladimir@uern.br\}
}

\begin{abstract}
With the significant growth of the Distance Education and its facilities offered by the technological apparatus, Virtual Environments of Learning have been appeared which add several technologies found on the Web, but it shows the need of tools for planning. In this way, will be shown a methodology for a learning process of a subject or Virtual Environments of Learning course that were idealized making sure that the concepts related with the Bloom's Revised Taxonomy and make the learning monitoring.

Resumo: Com o expressivo crescimento da Educação a Distância e as facilidades oferecidas pelo aparato tecnológico, tem surgido os Ambientes Virtuais de Aprendizagem que agregam várias tecnologias encontradas na Web, porém se evidencia a carência de ferramentas voltadas para o planejamento. Nesse sentido, será apresentada uma metodologia para o processo de planejamento de uma disciplina ou curso em Ambientes Virtuais de Aprendizagem que foi idealizado visando introduzir os conceitos relacionados com a Taxonomia Revisada de Bloom e possibilitar o acompanhamento da aprendizagem.
\end{abstract}

\section{Introdução}

A disseminação do uso de tecnologias em distintos ramos de atividades nos coloca frente às mudanças na cultura, na sociedade, na economia, na ciência e na dimensão educacional, em especial na Educação a Distância (EaD). De acordo com Schlemmer et. al. (2002), a utilização crescente das Tecnologias de Informação e Comunicação (TIC) tem contribuído para a disseminação de Ambientes Virtuais de Aprendizagem (AVA).

Em termos conceituais, Elliott (2004) afirma que os AVA são "um cenário de ensinar e aprender que projeta e realça a experiência de aprendizagem de estudantes" por meio de computadores e internet. Já para Araújo Júnior e Marquesi (2009) os AVA podem ser definidos, na perspectiva dos usuários, como ambientes que simulam os ambientes presenciais de aprendizagem com o uso das TIC.

A incorporação dos recursos tecnológicos na educação tem proporcionado reflexões sobre a forma de ensinar e aprender presencialmente ou a distância. De acordo com Yatsuda et. al. (2011) com o expressivo crescimento da Internet a EaD ganhou um novo impulso e consolidou-se como uma modalidade alternativa de aprendizagem que usa as TIC como possibilidade de interação no espaço virtual.

Com a popularização dos AVA, surgiu uma grande quantidade de ferramentas, com recursos pedagógicos, voltadas para o acompanhamento e avaliação da aprendizagem. Contudo, Lima e Fialho (2008) consideram que, embora exista uma 
quantidade significativa de ferramentas para os AVA, ainda há uma falta de instrumentos que possibilitem o planejamento da disciplina, coordenando $\mathrm{e}$ direcionando o uso desses elementos.

Como exemplificado em Masseto e Abreu (1980), o processo de ensino e aprendizagem é uma ação intencional e sistemática que, como todo o procedimento, precisa ser planejado e controlado adequadamente em todas as fases de execução. Nesse sentido, a presença de ferramentas nos AVA que auxilie o docente na elaboração do planejamento de ensino pode contribuir de forma significativa para o processo de ensino e aprendizagem.

É nesse enfoque, que este artigo aborda aspectos relevantes que estão relacionados com o desenvolvimento de ferramentas que auxilie o professor no planejamento do ensino em AVA e possibilite o acompanhamento da aprendizagem.

Além desta seção de introdução, este artigo está estruturado da seguinte forma: na Seção 2 descreve-se sobre a Taxonomia de Bloom; na Seção 3 expõe os Trabalhos Relacionados; na Seção 4 apresenta a Metodologia Proposta e por fim, na Seção 5 são apresentadas as Considerações Finais.

\section{Taxonomia de Bloom}

Uma das formas de inserir o planejamento no processo de ensino aprendizagem é trabalhar com objetivos educacionais [Bloom et. al., 1979]. O uso de objetivos educacionais permite ao professor planejar com base no que se espera do aluno e não apenas no conteúdo. Uma das teorias utilizadas para trabalhar com Objetivos Educacionais é a Taxonomia de Bloom [Bloom et. al., 1979].

Segundo Krathwohl (2002), Benjamim S. Bloom e uma comissão multidisciplinar de especialistas de várias universidades dos EUA criaram a Taxonomia buscando uma forma de facilitar o intercâmbio de questões de testes entre professores de várias universidades, e esse intercâmbio considerava a construção de um banco de teste, cada um deles avaliando o mesmo objetivo de aprendizagem. A Taxonomia de Bloom é um referencial para classificar afirmações sob as quais se espera que os alunos aprendam como resultado da instrução.

A Taxonomia de Bloom é constituída de três domínios: o cognitivo, afetivo e psicomotor, que caracterizam as habilidades, capacidades e atitudes que devem ser desenvolvidas no processo educacional [Pickard, 2007]. O domínio cognitivo trata das habilidades mentais e de pensamento que envolve lembrança, recognição, resolução de problemas e criatividade. Já o domínio afetivo abrange as áreas do sentimento e da emoção. Finalmente, o domínio psicomotor pertence à área das habilidades físicas e manuais [Bloom et. al., 1979]. Neste trabalho será abordado somente o Domínio Cognitivo.

O domínio cognitivo da Taxonomia de Bloom é um modelo multicamadas que classifica o pensamento de acordo com seis níveis de complexidade cognitiva. As categorias do domínio cognitivo são: Conhecimento, Compreensão, Aplicação, Análise, Síntese e Avaliação. Com exceção de Aplicação, as demais foram divididas em subcategoria. Estas categorias são ordenadas da mais simples para a mais complexa, definindo uma hierarquia cumulativa, onde uma categoria mais simples é pré-requisito para uma categoria mais complexa [Bloom et. al., 1979].

Segundo Filatro (2009), a Taxonomia de objetivos educacionais desenvolvida por Bloom et. al. (1979), influenciou significativamente a sistemática de planejamento pedagógico, na medida em que criou uma linguagem comum e padronizada para identificar e classificar as atividades educacionais. 
Para Anderson et. al. (2001), o caráter histórico da Taxonomia original pode ser evidenciado partindo da estrutura desta, levando-se em conta as teorias vigentes na época nas áreas de educação e psicologia. Neste sentido, uma nova taxonomia se faz necessária para que se possa considerar as indicações e prescrições das pesquisas mais recentes nestas áreas do conhecimento [Bümen, 2007].

De acordo com Anderson (2005), entre 1995 e 2000, um grupo de educadores trabalhou para revisar a Taxonomia de Bloom, o que deu origem a Taxonomia Revisada de Bloom. Essa Taxonomia Revisada foi estruturada com base em definições cuidadosas para a Dimensão do Conhecimento (substantivos - o que) e a Dimensão Processo Cognitivo (verbos - como) [Krathwohl, 2002]. A Figura 1 ilustra essa nova divisão.

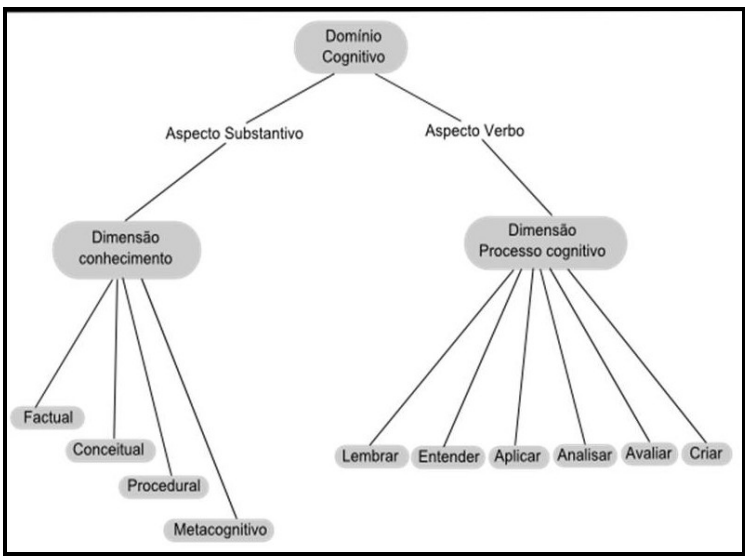

Figura 1: Dimensões do Domínio Cognitivo da Taxonomia Revisada de Bloom.

Como consequência, a Taxonomia Revisada contém duas dimensões: "Dimensão Processo Cognitivo" e "Dimensão do Conhecimento". Isso originou um novo modelo de utilização que tem como estrutura uma tabela bidimensional, conforme apresentado pela Tabela 1 .

Tabela 1: Tabela bidimensional da Taxonomia Revisada de Bloom. Fonte: [Anderson et. al. 2001, p. 28].

\begin{tabular}{|l|c|c|c|c|c|c|}
\hline \multirow{2}{*}{$\begin{array}{c}\text { Dimensão do } \\
\text { Conhecimento }\end{array}$} & \multicolumn{5}{|c|}{ Dimensão dos Processos Cognitivos } \\
\cline { 2 - 7 } & 1 & 2 & 3 & 4 & 5 & 6 \\
Aembrar & Entender & Aplicar & Analisar & Avaliar & Criar \\
\hline A. Efetivo/factual & & & & & & \\
\hline B. Conceitual & & & & & & \\
\hline C. Procedural & & & & & & \\
\hline D. Metacognitivo & & & & & & \\
\hline
\end{tabular}

Na nova estrutura, a Dimensão do Conhecimento pertence à coluna vertical e a Dimensão Processo Cognitivo à coluna horizontal. Nas células, formadas pela intersecção das dimensões, são inseridos os objetivos.

Embora a Taxionomia Revisada mantenha o mesmo design da original, onde as categorias da Dimensão Processo Cognitivo são ordenadas da mais simples para a mais complexa, a restrição de uma "hierarquia cumulativa" foi removida. Portanto a Taxionomia Revisada é flexível, pois possibilita considerar a interpolação das categorias do processo cognitivo quando necessário.

A Dimensão do Conhecimento consiste em quatro tipos de conhecimentos: factual, conceitual, procedimental e metacognitivo. Para Anderson et. al., (2001) e Krathwohl (2002), a ordem das categorias deve ser respeitada, pois se considera que não 
há como estimular ou avaliar o conhecimento metacognitivo sem anteriormente ter adquirido todos os anteriores.

De acordo com Wilson (2006) o professor pode utilizar a tabela bidimensional da Taxonomia Revisada de Bloom para controlar quais os níveis da Dimensão Processo cognitivo estão sendo exigidos dos alunos, bem como as categorias da Dimensão do Conhecimento.

Nesse contexto, será apresentado um exemplo prático na Tabela 02. Assim a montagem da tabela deve ser realizada a partir das definições dos objetivos educacionais. Segundo Ferguson (2002) o professor da disciplina de história desenvolveu o planejamento utilizando a Taxonomia Revisada de Bloom. O curso intitulado "Civilização Ocidental" foi desenvolvido com base nas normas do Sul do estado de Carolina, e em um dos módulos, os alunos deveriam estudar o conteúdo histórico da Revolução Francesa. Portanto os objetivos foram assim descritos:

Ao final do módulo, os alunos deverão ser capazes de:

1. Lembrar dos principais personagens, eventos, e as datas relacionadas com a Revolução Francesa;

2. Sintetizar e ser capaz de explicar as causas da Revolução Francesa;

3. Comparar as três fases da Revolução Francesa.

Tabela 2 - Preenchimento da Tabela bidimensional. Fonte:Adaptada de (Ferguson, 2002).

\begin{tabular}{|l|c|c|c|c|c|c|}
\hline \multirow{2}{*}{$\begin{array}{c}\text { Dimensão do } \\
\text { Conhecimento }\end{array}$} & \multicolumn{6}{|c|}{ Dimensão dos Processos Cognitivos } \\
\cline { 2 - 7 } & Lembrar & Entender & Aplicar & Analisar & Avaliar & Criar \\
\hline Efetivo/factual & Objetivo1 & & & & & \\
\hline Conceitual & & $\begin{array}{l}\text { Objetivo2 } \\
\text { Objetivo3 }\end{array}$ & & & & \\
\hline Procedural & & & & & & \\
\hline Metacognitivo & & & & & & \\
\hline
\end{tabular}

É importante salientar que, conforme mencionado anteriormente, a ordem da Dimensão do Conhecimento deve ser respeitada de forma hierárquica, entretanto não há nenhum problema na ordem dos objetivos inseridos na Dimensão Processo Cognitivo.

\section{Trabalhos Relacionados}

$\mathrm{Na}$ literatura, vários trabalhos fazem referências ao uso de Ambientes Virtuais de Aprendizagem na tentativa de melhorar o desempenho dos estudantes.

Albuquerque e Leite (2008) apresentam um projeto que desenvolveram para uma disciplina de um curso de graduação em Ciências Biológicas, utilizando um ambiente virtual de aprendizagem como recurso didático. Nesse trabalho foi ratificado que a tecnologia educativa de AVA se mostrou ser uma ferramenta potencial para complementar o ensino de ciências realizado em sala de aula e em laboratórios, bem como um recurso da $\mathrm{EaD}$ motivador e inovador para o professor e para o aluno. Os autores afirmam que foi evidenciado um aprendizado dinâmico e participativo, contribuindo para a formação acadêmica e cidadã dos alunos do ensino superior.

Lima e Fialho (2011) apresentam duas ferramentas pedagógicas, o Mapa de Conteúdos (MC) e o Mapa de Dependências (MD), que inserem o planejamento com 
base em objetivos educacionais e possibilitam trabalhar o processo de ensinoaprendizagem nos AVA.

As ferramentas MC e MD foram implementadas no Ambiente Moodle [Moodle, 2013] através da definição de um novo tipo de curso, que ao ser escolhido, direciona o professor no planejamento da disciplina.

A ferramenta Mapa de Conteúdos é baseada na Aprendizagem Significativa [Ausubel, 1976] e nos Mapas Conceituais [Novak; Cañas, 2006] e possibilita apresentar a disciplina ou curso por meio de uma visualização gráfica dos conteúdos e das relações existentes entre os mesmos. Já a ferramenta Mapa de Dependências que tem como aporte teórico a Taxonomia de Bloom [Bloom et. al., 1979] possibilita ao professor definir em cada conteúdo, além do objetivo educacional que se almeja, as capacidades e habilidades necessárias para se atingir o objetivo proposto.

Assim, embora as ferramentas pedagógicas MC e MD contribuam de forma significativa para auxiliar o docente no planejamento de uma disciplina ou curso em Ambientes Virtuais de Aprendizagem, as suas metodologias de criação estão baseadas em um processo de interação distintos, desenvolvidas em módulos separados, o que possibilita o professor usar apenas um dos módulos, o que pode gerar um problema ao processo de planejamento e acompanhamento da aprendizagem.

Dessa forma, verifica-se a necessidade de modificar a metodologia de criação do MC e do MD de forma a integrar o processo de planejamento em uma única metodologia possibilitando o professor definir os conteúdos, os objetivos educacionais e em seguida gerar o MC e MD.

Nesse enfoque, na próxima seção será apresentada a proposta metodológica com base na Taxonomia Revisada de Bloom [Anderson et. al., 2001], que através das duas dimensões do Domínio Cognitivo: Dimensão do Conhecimento e Dimensão Processo Cognitivo, possibilita integrar o processo de planejamento em uma única metodologia.

\section{Metodologia Proposta}

De acordo com Litto e Formiga (2009) os modelos de educação a distância vigentes incluem em sua formulação estruturas administrativas voltadas para ferramentas on-line, modelo pedagógico adaptado aos sistemas virtuais de comunicação e ferramentas tecnológicas próprias.

Dentre os desafios da EaD destaca-se a necessidade de implantação de mecanismos para o planejamento do processo ensino-aprendizagem, que coordenem esses recursos, e que auxilie o professor no processo de planejamento de ensino e possibilite o acompanhamento da aprendizagem.

Uma das maneiras de inserir o planejamento no processo de ensino aprendizagem é trabalhar com a Taxonomia Revisada de Bloom [Anderson et. al., 2001], que através da Dimensão do Conhecimento e Dimensão Processo Cognitivo, possibilita o professor definir os conteúdos e os objetivos educacionais da unidade, disciplina ou curso.

Conforme mencionado na seção 2, na nova estrutura da Taxonomia Revisada de Bloom, a Dimensão do Conhecimento está diretamente relacionada ao conteúdo e consiste em quatro tipos de conhecimentos: factual, conceitual, procedural e metacognitivo. De acordo com Anderson et. al., (2001) e Krathwohl (2002) a ordem das categorias deve ser respeitada, pois se considera que não há como estimular ou avaliar o conhecimento metacognitivo sem anteriormente ter adquirido todos os anteriores.

De acordo com Anderson et. al., (2001) e Krathwohl (2002) a Dimensão Processo Cognitivo abrange as seis categorias da Taxonomia original de Bloom, porém 
todas as categorias foram renomeadas para verbos, para ajustar com a forma como frequentemente docentes utilizam-na para deliberar seus objetivos durante a fase de planejamento de um módulo, disciplina ou curso.

Nesse enfoque, a metodologia proposta neste trabalho está voltada para o planejamento do ensino e aprendizagem, na perspectiva de auxiliar o professor no processo de planejamento de uma disciplina ou curso em Ambientes Virtuais de Aprendizagem que servem de apoio a Educação a Distância e ao ensino presencial em sala de aula. Para isso a metodologia é dividida em quatro fases: Trabalhar a Dimensão do Conhecimento; Trabalhar a Dimensão Processo Cognitivo; Gerar o Mapa de Conteúdos e Gerar o Mapa de Dependências. O Algoritmo 01 ilustra as fases da metodologia.

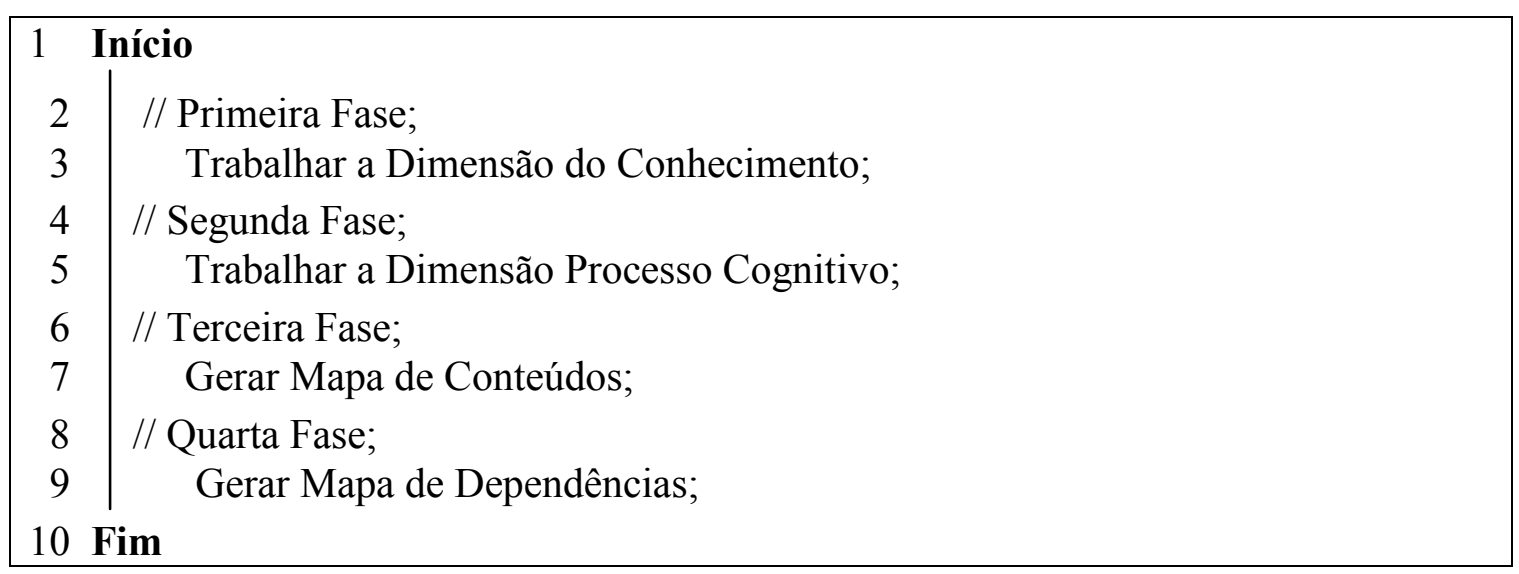

Algoritmo 01: Fases da metodologia de planejamento

Na primeira fase, representada pela terceira linha do Algoritmo 01, o objetivo da metodologia é, com base na Dimensão do Conhecimento da Taxonomia Revisada de Bloom, tornar as informações sobre o conteúdo da disciplina mais significativo para o aluno. Nesse sentido o docente torna evidente a definição dos conteúdos e as relações hierárquicas existentes entre os conteúdos, de acordo com a Dimensão do Conhecimento da Taxonomia Revisada de Bloom.

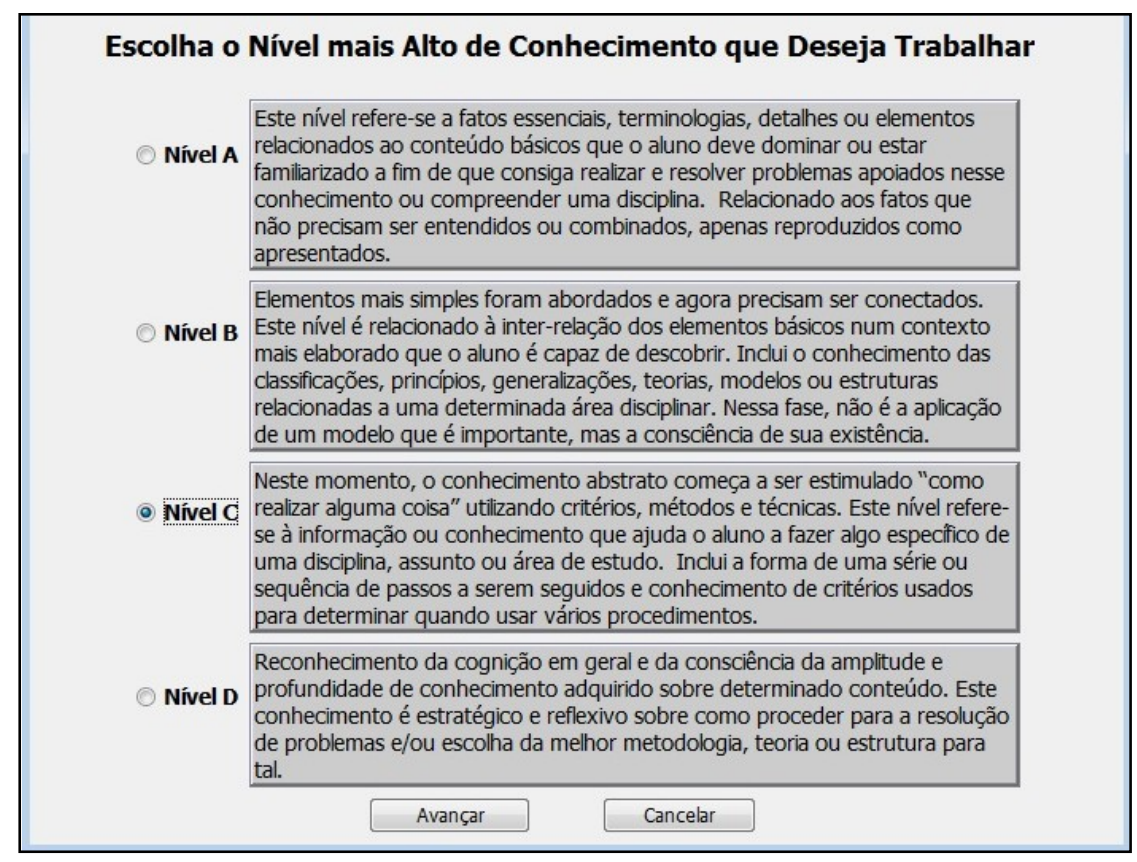

Figura 2: Tipos de Dimensões do Conhecimento. Fonte: Adaptada de [Anderson et. al. 2001 e de Krathwohl 2002]. 
Nesse contexto, para auxiliar o professor no momento que ele irá relacionar o conteúdo com o tipo de conhecimento, será exibido a Figura 2 que apresenta os quatro níveis da Dimensão do Conhecimento através das letras A, B, C, e D.

O Conhecimento Factual, primeira categoria da Dimensão do Conhecimento e o nível mais baixo, está representado pela letra A. O segundo nível é a categoria Conhecimento Conceitual representado pela letra B. Já o terceiro nível é a categoria Conhecimento Procedural, representado pela letra $\mathrm{C}$ e por fim, a letra D representa a quarta categoria Conhecimento Metacognitivo, considerada o quarto e mais alto nível da Dimensão do Conhecimento.

Dessa forma, o docente deve sempre informar para cada conteúdo inserido o nível mais alto de conhecimento que deseja trabalhar, para isso o professor ao escolher o nível $\mathrm{C}$ tem que informar o conteúdo para o nível $\mathrm{C}$, bem como para os níveis anteriores, respectivamente os níveis $\mathrm{B}$ e $\mathrm{A}$.

Após a definição dos conteúdos e a relação deles com os níveis da Dimensão do Conhecimento, a próxima fase da metodologia é definir os objetivos educacionais para os conteúdos que foram informados pelo docente. Nessa etapa o professor por meio da Dimensão Processo Cognitivo escolhe o verbo e descreve textualmente o objetivo educacional.

Para auxiliar o professor na identificação das categorias do processo cognitivo será disponibilizado para o professor as categorias da Dimensão Processo Cognitivo e os verbos que estão associados às respectivas categorias, de acordo com a Tabela 3.

Tabela 3: Categorias do Processo Cognitivo e Processos Cognitivos relacionados. Fonte: [Anderson et. al. 2001, p. 31, 67-68].

\begin{tabular}{|l|l|l|l|l|l|}
\hline \multicolumn{6}{|c|}{ Dimensão Processo cognitivo } \\
\hline Lembrar & \multicolumn{1}{|c|}{ Entender } & \multicolumn{1}{|c|}{ Aplicar } & \multicolumn{1}{|c|}{ Analisar } & \multicolumn{1}{|c|}{ Avaliar } & \multicolumn{1}{c|}{ Criar } \\
\hline Reconhecer, & Interpretar, & Executar, & Diferenciar & Verificar, & Gerar, \\
Identificar, & Clarificar, & Fazer, & Discriminar, & Coordenar, & Hipotetizar, \\
Recordar, & Parafrasear, & Implementar, & Distinguir, & Detectar, & Planejar, \\
Lembrar. & Representar, & Usar. & Focar, & Monitorizar, & Desenhar, \\
& Traduzir, & & Selecionar, & Testar, & Produzir, \\
& Exemplificar, & & Organizar, & Criticar, & Construir. \\
& Ilustrar & & Integrar, & Julgar. & \\
& Representar, & & Desenhar, & & \\
& Classificar, & & Estruturar, & & \\
& Categorizar, & & Encontrar, & & \\
& Agrupar, Resumir, & & Coerenciar, & & \\
& Sintetizar, & & Atribuir, & & \\
& Generalizar, & & Descontruir. & & \\
& Inferir, & & & & \\
& Concluir, & & & & \\
& Extrapolar, & & & & \\
& Interpolar, & & & & \\
& Prever, & & & & \\
& Comparar, & & & & \\
& Contrastar, & & & & \\
& Mapear, & & & \\
& Corresponder, & & & & \\
& Explicar, & & & & \\
& Construir modelos. & & & & \\
\end{tabular}

Após o professor descrever textualmente o primeiro Objetivo Educacional, repetirá o mesmo procedimento para descrever os demais Objetivos, referente a cada 
conteúdo que foi relacionado hierarquicamente com os níveis da Dimensão do Conhecimento.

A montagem da tabela bidimensional da Taxonomia Revisada de Bloom deve iniciar-se a partir das definições dos objetivos específicos (OE) da unidade, disciplina ou curso. Essa tabela deve ser utilizada com o intuito de melhor definir os objetivos educacionais, com vista a contribuir para um melhor planejamento de ensino.

Nesse contexto, será apresentado um exemplo prático na Tabela 4. Assim, os objetivos proposto no exemplo são para uma disciplina hipotética, que será denominada Ciências Naturais dentro da qual é tratado o tópico água.

Essa disciplina normalmente aborda outros tópicos e deverá ser dividida em módulos e, num deles, os estudantes deverão entender os conceitos e a importância da conservação da água. Portanto os objetivos poderiam ser assim descritos:

Ao final da unidade, os alunos deverão ser capazes de:

1. Identificar a distribuição de água no planeta e os fatores naturais e sociais que interferem na sua abundância e escassez, tendo em vista o consumo humano;

2. Reconhecer as diferentes etapas e processos que constituem o ciclo da água na natureza e avaliar repercussões das alterações nele promovidas pelas atividades humanas.

3. Usar exemplos de lugares ou regiões geográficas do país e do mundo que necessitam de água para explicar a ideia de que todos não têm acesso;

4. Distinguir práticas e situações que comprometem a disponibilidade de água no Brasil e no mundo e examinar propostas para o seu uso sustentável;

5. Planejar e promover ações na escola e na comunidade que contribuam para preservar os recursos hídricos e evitar usos inadequados da água.

Tabela 4 - Montagem da Tabela bidimensional

\begin{tabular}{|l|c|c|c|c|c|c|}
\hline \multirow{2}{*}{$\begin{array}{c}\text { Dimensão do } \\
\text { Conhecimento }\end{array}$} & \multicolumn{5}{|c|}{ Dimensão dos Processos Cognitivos } \\
\cline { 2 - 7 } & Lembrar & Entender & Aplicar & Analisar & Avaliar & Criar \\
\hline Efetivo/factual & OE 1 & OE 2 & & & & \\
\hline Conceitual & & & OE 3 & OE 4 & & \\
\hline Procedural & & & & & & OE 5 \\
\hline Metacognitivo & & & & & & \\
\hline
\end{tabular}

Ao observar a Tabela 4, percebe-se que há colunas em branco e quais foram os níveis da Dimensão do Conhecimento exigidos dos aprendizes, bem como quais categorias da Dimensão Processo cognitivo. Nesse contexto, o professor é levado a observar as colunas em branco e pode fazer uma análise e reavaliação dos objetivos proposto e isso pode contribuir para melhorar o seu planejamento de ensino.

Por fim, na terceira e quarta fases da metodologia serão gerados, respectivamente, o Mapa de Conteúdos e o Mapa de Dependências. O MC será gerado a partir dos conteúdos inseridos pelo docente na Dimensão do Conhecimento e o MD por meio das duas dimensões da tabela de dupla entrada, relacionado aos objetivos definidos pelo professor. 


\section{Considerações Finais}

Com a explosão da internet, rapidamente os AVA destinados ao uso educativo foram incorporados ao ciberespaço. Dessa forma, a aprendizagem é cada vez mais apoiada pelas TIC e pelas interações por elas geradas. Nesse enfoque, as tecnologias digitais e em rede estão cada vez mais incorporadas à sala de aula, seja na modalidade presencial, hibrida ou a distância.

Para Klosouski e Reali (2008) a aprendizagem na atualidade é entendida dentro de uma visão construtivista como um resultado do esforço de encontrar significado ao que se está aprendendo. As autoras dizem que se realmente há a preocupação com a aprendizagem, deve-se questionar se a forma como se planeja tem em mente também o ensino, ou seja, deve haver uma co-relação entre ensino-aprendizagem.

No contexto da $\mathrm{EaD}$, o planejamento torna-se um instrumento importante. $\mathrm{Na}$ fase de preparação do planejamento de ensino, é essencial a formulação dos objetivos educacionais. Segundo Martins (1990), talvez seja uma das decisões mais complexas a ser feita por parte do educador, pois ela norteará todo o processo de planejamento, plano de ensino, conteúdos, estratégias e o processo de avaliação a serem utilizadas.

Nesse contexto, este trabalho propõe o desenvolvimento de uma metodologia voltada para o processo de ensino e aprendizagem, na perspectiva de auxiliar o professor no planejamento de uma disciplina ou curso em Ambientes Virtuais de Aprendizagem com base na Taxonomia Revisada de Bloom [Anderson et. al. 2001].

Para isso, foi apresentado um modelo visando integrar a metodologia de criação das ferramentas pedagógicas MC e MD [Lima e Fialho 2011] de forma a agregar o processo de planejamento em uma única metodologia, o que possibilita o educador definir os conteúdos e relaciona-los hierarquicamente com os níveis da Dimensão do Conhecimento e definir os objetivos educacionais utilizando a categorias da Dimensão Processo Cognitivo, para com isso gerar o $\mathrm{MC}$ e o MD, além de inserir conteúdos e atividades que serão desenvolvidas pelos alunos em um AVA.

\section{Referências Bibliográficas}

Albuquerque, R. C.; Leite, S. Q. M. (2008) Uso de ambientes virtuais de aprendizagem como estratégia educacional complementar de ensino de ciências. CINTEDUFRGS Novas Tecnologias na Educação. V. $6 \mathrm{~N}^{\circ} 1$.

Anderson, L. W.; Krathwohl, D. R.; Airasian, P. W.; Cruikshank, K. A.; Mayer, R. E.; Pintrich, P. R.; Raths, J.; Wittrock, M. C. (2001) "A taxonomy for learning, teaching, and assessing: a revision of Bloom's Taxonomy of Educational Objectives". Longman: New York.

Anderson, L. W. (2005) "Objectives, evaluation, and the improvement of education". Studies in Educational Evaluation, Amsterdan, v. 31, n. 2/3, p. 102-113.

Araújo Júnior, C. F.; Marquesi, S. C. (2009) "Atividades em ambientes virtuais de aprendizagem: parâmetros de qualidade", In: Litto, F. M.; Formiga, M. M. M. Educação a Distância: o estado da arte. São Paulo: Pearson Education do Brasil, p.358-368.

Ausubel, D. P. (1976) "Psicología educativa: un punto de vista cognoscitivo". Roberto Helier Domínguez. México: Trillas.

Bloom, B. S.; Engelhart, M. D.; Furst, E. J.; Hill, W. H.; Krathwohl, D. R. (1979) "Taxonomia de objetivos educacionais - domínio cognitivo". Globo: Porto Alegre - RS. 
Bümen, T. N. (2007) Effects of original versus revised Bloom,s Taxonomy on lesson planning skills: a turkish study among pre-service teachers. Review of Education, $n^{\circ} .53$, p. $439-455$.

Elliott, H. (2004) Virtual Learning Environments: Using Online Course Management Systems to Implement Constructivism in Learning at the Secondary Level. Disponível em: < https://moodle.org/other/dEntremont_Final_Paper.pdf $>$ Acesso em Fev de 2011.

Ferguson, C. (2002) The Revised Taxonomy and Thematic Units In: Theory into Practice, n. 41, v. 4, p. 238-243.

Filatro, A. (2009) "As teorias pedagógicas fundamentais em EAD”. In: Litto, Fredric Michael; Formiga, Manuel Marcos Maciel. Educação a Distância: o estado da arte. São Paulo: Pearson Education do Brasil, p.96-104. Krathwohl, D. R. A revision of bloom's taxonomy: an overview, In: Theory into Practice, n. 41, v. 4, p. 212-218, 2002.

Klosouski S. S.; Reali K. M. (2008) Planejamento de ensino como ferramenta básica do processo ensino- aprendizagem. UNICENTRO - Revista Eletrônica Lato Sensu. Ed.5. ISSN: 1980-6116.

Krathwohl, D. R. (2002) A revision of bloom's taxonomy: an overview, In: Theory into Practice, n. 41, v. 4, p. 212-218.

Lima, R. W.; Fialho, S. V. (2008) "Mapa de Dependências: uma ferramenta para aplicação da Taxonomia de Bloom na Educação a Distância”. In: XIX Simpósio Brasileiro de Informática na Educação, Fortaleza - CE.

Lima, R. W. ; Fialho, S. V. (2011) Mapa de Conteúdos e Mapa de Dependências: ferramentas para um planejamento com base em objetivos educacionais. Revista de Exatas e Tecnológica - RETEC, v. 2, p. 10.

Litto, F. M.; Formiga, M. (2009) Educação a Distância, Pearson Brasil, São Paulo.

Martins, J. P. (1990) “Didática Geral”. São Paulo: Atlas, 1990. Masetto, M. T.; Abreu, M C. O professor universitário em aula: prática e princípios teóricos. São Paulo: MG Associados Ltda.

Masetto, M. T.; Abreu, M C. (1980) O professor universitário em aula: prática e princípios teóricos. São Paulo: MG Associados Ltda.

Moodle. Moodle.org: open-souce community-based tools for learning. 2013. Disponível em: <http://www.moodle.org > Acesso em Jan de 2013.

Novak, J. D.; Cañas, A. J. (2006) "The theory undelying concept maps and how to construct them". Techinical Report IHCM CmapTools 2006-1, Florida Institute for Human and Machine Cognition.

Pickard, M. J. (2007) The new Bloom's Taxonomy: an overview for family and consumer sciences. Journal of Family and Consumer Sciences Education, Vol. 25, No. 1.

Schlemmer, E. et. al. ( 2002), AVA: Um Ambiente Virtual Baseado em Comunidades. In XIII Simpósio Brasileiro de Informática na Educação (SBIE), São Leopoldio UNISINOS. P. 31-38.

Wilson, Leslie O., Ed. D. (2006). Anderson and Krathwohl, Beyond Bloom. University of Wisconsin-Stevens Point. Retrieved November 20, 2006 from. Disponível em: $<$ http://www.uwsp.edu/education/lwilson/curric/newtaxonomy.htm $>$ Acesso Jan 2013.

Yatsuda, G. A.; Schiavoni, F. L.; Medeiros Filhos, D. A.; Toffolo, R. B. G. (2011) TAW -Treinamento Auditivo pela WEB: Ensino Musical a Distância. In: XXII Simpósio Brasileiro de Informática na Educação - SBIE. Aracajú - SE. p. 771-780. 